\title{
Symmetries and Invariant Differential Pairings ${ }^{\star}$
}

\author{
Michael G. EASTWOOD
}

Department of Mathematics, University of Adelaide, SA 5005, Australia

E-mail: meastwoo@member.ams.org

Received November 14, 2007; Published online November 23, 2007

Original article is available at http://www.emis.de/journals/SIGMA/2007/113/

\begin{abstract}
The purpose of this article is to motivate the study of invariant, and especially conformally invariant, differential pairings. Since a general theory is lacking, this work merely presents some interesting examples of these pairings, explains how they naturally arise, and formulates various associated problems.
\end{abstract}

Key words: conformal invariance; differential pairing; symmetry

2000 Mathematics Subject Classification: 53A30; 58J70; 53A20

\section{Introduction}

The Lie derivative is an extremely familiar operation in differential geometry. Given a smooth vector field $V$ and tensor field $\phi$, the Lie derivative $\mathcal{L}_{V} \phi$ is certainly an intrinsic or invariant construction. In practise, we can take this to mean that the result is independent of any local coördinate formula that may be used. Alternatively, we may employ an arbitrary torsion-free affine connection $\nabla$ to write explicit formulae such as

$$
\phi_{b c} \stackrel{\mathcal{L}_{V}}{\longmapsto} V^{a} \nabla_{a} \phi_{b c}+\left(\nabla_{b} V^{a}\right) \phi_{a c}+\left(\nabla_{c} V^{a}\right) \phi_{b a}
$$

checking that the result is independent of the connection used. In this formula, the indices are 'abstract indices' in the sense of Penrose [18]. It follows that the expression is coördinate-free whilst its freedom from choice of connection follows immediately from the formulae

$$
\widehat{\nabla}_{a} V^{c}=\nabla_{a} V^{c}+\Gamma_{a b}^{c} V^{b} \quad \text { and } \quad \widehat{\nabla}_{a} \phi_{b c}=\nabla_{a} \phi_{b c}-\Gamma_{a b}{ }^{d} \phi_{d c}-\Gamma_{a c}^{d} \phi_{b d},
$$

capturing all possible torsion-free connections via the choice of tensor $\Gamma_{a b}{ }^{c}=\Gamma_{b a}{ }^{c}$. For further details on this point of view, see [18].

Writing the Lie derivative as $\mathcal{L}_{V} \phi$ suggests that we are regarding it as a linear differential operator acting on the tensor field $\phi$ for a fixed choice of vector field $V$. However, it is clear from expressions such as (1) that we may equally well fix $\phi$ and regard the result as a linear differential operator acting on vector fields $V$. Sometimes, this is the natural viewpoint. For example, if $\phi_{a b}$ is symmetric then we may rewrite (1) as

$$
\left(\mathcal{L}_{V} \phi\right)_{b c}=\phi_{b c} \nabla_{a} V^{c}+\phi_{a c} \nabla_{b} V^{c}+\left(\nabla_{c} \phi_{a b}\right) V^{c}
$$

and now if $\phi_{a b}$ is a metric and we choose $\nabla_{a}$ to be its Levi-Civita connection, then

$$
\mathcal{L}_{V} \phi=0 \Longleftrightarrow \nabla_{a} V_{b}+\nabla_{b} V_{a}=0
$$

${ }^{\star}$ This paper is a contribution to the Proceedings of the Seventh International Conference "Symmetry in Nonlinear Mathematical Physics" (June 24-30, 2007, Kyiv, Ukraine). The full collection is available at http://www.emis.de/journals/SIGMA/symmetry2007.html 
which is the usual way of viewing the Killing equation on a Riemannian manifold. Of course, the best way of viewing $\mathcal{L}_{V} \phi$ is as a bilinear differential operator or as a differential pairing. It is invariantly defined on any manifold and is an example of the subject matter of this article.

One can easily imagine other expressions along the lines of the right hand side of (1) that turn out to be independent of choice of connection. Restricting the choice of connection leads to a greater range of expressions that might be invariant. In the next two sections we shall find examples that are invariant when the connections are deemed to be Levi-Civita connections for metrics taken from a fixed conformal class.

\section{First order symmetries}

Suppose $\mathrm{E}: E \rightarrow F$ is a linear differential operator between vector bundles $E$ and $F$. A linear differential operator $\mathcal{D}: E \rightarrow E$ is said to be a symmetry of $\mathrm{E}$ if and only if

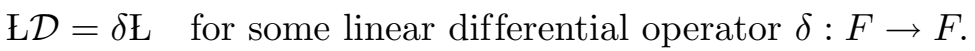

When $\mathrm{E}$ is the Laplacian on $\mathbb{R}^{3}$ (and the bundles $E$ and $F$ are trivial), its first order symmetries are well-known [17]. Specifically, they comprise an 11-dimensional vector space spanned by the following

$$
\begin{aligned}
& f \mapsto f, \quad f \mapsto \frac{\partial f}{\partial x}, \quad f \mapsto \frac{\partial f}{\partial y}, \quad f \mapsto \frac{\partial f}{\partial z}, \quad f \mapsto x \frac{\partial f}{\partial x}+y \frac{\partial f}{\partial y}+z \frac{\partial f}{\partial z}, \\
& f \mapsto x \frac{\partial f}{\partial y}-y \frac{\partial f}{\partial x}, \quad f \mapsto\left(x^{2}-y^{2}-z^{2}\right) \frac{\partial f}{\partial x}+2 x y \frac{\partial f}{\partial y}+2 x z \frac{\partial f}{\partial z}+x f, \\
& f \mapsto y \frac{\partial f}{\partial z}-z \frac{\partial f}{\partial y}, \quad f \mapsto\left(y^{2}-z^{2}-x^{2}\right) \frac{\partial f}{\partial y}+2 y z \frac{\partial f}{\partial z}+2 y x \frac{\partial f}{\partial x}+y f, \\
& f \mapsto z \frac{\partial f}{\partial x}-x \frac{\partial f}{\partial z}, \quad f \mapsto\left(z^{2}-x^{2}-y^{2}\right) \frac{\partial f}{\partial z}+2 z x \frac{\partial f}{\partial x}+2 z y \frac{\partial f}{\partial y}+z f,
\end{aligned}
$$

where $x, y, z$ are the usual Euclidean coördinates on $\mathbb{R}^{3}$. Following [10], it is convenient to rewrite the general first order symmetry as

$$
f \mapsto V^{a} \nabla_{a} f+\frac{1}{6}\left(\nabla_{a} V^{a}\right) f+C f,
$$

where $V^{a}$ is an arbitrary vector field of the form

$$
V^{a}=-s^{a}-m_{b}^{a} x^{b}+\lambda x^{a}+r_{b} x^{b} x^{a}-\frac{1}{2} x_{b} x^{b} r^{a}
$$

and $C$ is an arbitrary constant. In fact, vector fields of the form (4) on $\mathbb{R}^{n}$ are precisely the conformal Killing fields, i.e. the solutions of the equation

$$
\nabla_{a} V_{b}+\nabla_{b} V_{a}=\frac{2}{n} g_{a b} \nabla^{c} V_{c}
$$

where $g_{a b}$ is the usual Euclidean metric on $\mathbb{R}^{n}$. More generally, on $\mathbb{R}^{n}$ the first order symmetries of the Laplacian may be written as $f \mapsto \mathcal{D}_{V} f+C f$, where

$$
\mathcal{D}_{V} f \equiv V^{a} \nabla_{a} f+\frac{n-2}{2 n}\left(\nabla_{a} V^{a}\right) f
$$

for an arbitrary conformal Killing field $V^{a}$. This expression certainly resembles the Lie derivative and we now make this precise as follows. If $\phi_{b c \cdots d e}$ is an $n$-form, then

$$
0=(n+1)\left(\nabla_{[a} V^{a}\right) \phi_{b c \cdots d e]}=\left(\nabla_{a} V^{a}\right) \phi_{b c \cdots d e}-\left[\left(\nabla_{b} V^{a}\right) \phi_{a c \cdots d e}+\cdots+\left(\nabla_{e} V^{a}\right) \phi_{b c \cdots d a}\right],
$$


where we are following [18] in employing square brackets to denote the result of skewing over the indices they enclose and also noting that a skew tensor with $n+1$ indices on $\mathbb{R}^{n}$ necessarily vanishes. Hence, the Lie derivative on $n$-forms simplifies:

$$
\begin{aligned}
\mathcal{L}_{V} \phi_{b c \cdots d e} & =V^{a} \nabla_{a} \phi_{b c \cdots d e}+\left(\nabla_{b} V^{a}\right) \phi_{a c \cdots d e}+\cdots+\left(\nabla_{e} V^{a}\right) \phi_{b c \cdots d a} \\
& =V^{a} \nabla_{a} \phi_{b c \cdots d e}+\left(\nabla_{a} V^{a}\right) \phi_{b c \cdots d e} .
\end{aligned}
$$

Thus, if $h$ is a section of the bundle $\Lambda^{n}$ of $n$-forms, then

$$
\mathcal{L}_{V} h=V^{a} \nabla_{a} h+\left(\nabla_{a} V^{a}\right) h .
$$

Therefore, the expression (6) coincides with the Lie derivative if $f$ is interpreted as a section of the line bundle $\left(\Lambda^{n}\right)^{(n-2) / 2 n}$ (supposing that the manifold is orientable so that such fractional powers are allowed). In particular, we deduce that $\left[\mathcal{D}_{V}, \mathcal{D}_{W}\right]=\mathcal{D}_{[V, W]}$, as is also readily verified by direct computation. Also we conclude that, with this interpretation, the differential pairing $\mathcal{D}_{V} f$ between $V^{a}$ and $f$ is invariant in the sense discussed for Lie derivative in Section 1.

\section{Conformal geometry}

An alternative viewpoint on these matters is as follows. If $\nabla_{a}$ is the Levi-Civita connection for a metric $g_{a b}$, then the Levi-Civita connection $\widehat{\nabla}_{a}$ for the conformally related metric $\widehat{g}_{a b}=\Omega^{2} g_{a b}$ is given on 1 -forms by

$$
\widehat{\nabla}_{a} \phi_{b}=\nabla_{a} \phi_{b}-\Upsilon_{a} \phi_{b}-\Upsilon_{b} \phi_{a}+\Upsilon^{c} \phi_{c} g_{a b},
$$

where $\Upsilon_{a}=\nabla_{a} \log \Omega$ and $\Upsilon^{c}=g^{c d} \Upsilon_{d}$. Thus, associated with a conformal class of metrics we have a restricted supply of torsion-free connections related by formulae such as (2) where

$$
\Gamma_{a b}{ }^{c}=\Upsilon_{a} \delta_{b}{ }^{c}+\Upsilon_{b} \delta_{a}^{c}-g_{a b} \Upsilon^{c}
$$

for closed 1-forms $\Upsilon_{a}$. It is convenient introduce a line bundle $L$ on a general conformal manifold as follows. A choice of metric $g_{a b}$ in the conformal class trivialises $L$, allowing us to view a section of $L$ as a function $f$. We decree, however, that viewing the same section by means of the trivialisation due to $\widehat{g}_{a b}=\Omega^{2} g_{a b}$ gives us the function $\widehat{f}=\Omega f$. More generally, sections of the bundle $L^{w}$ transform by $\widehat{f}=\Omega^{w} f$ and are called conformal densities of conformal weight $w$. Since

$$
\nabla_{a} \widehat{f}=\nabla_{a}\left(\Omega^{w} f\right)=\Omega^{w}\left(\nabla_{a} f+w \Upsilon_{a} f\right)
$$

we may view $L^{w}$ as equipped with a family of connections related by

$$
\widehat{\nabla}_{a} f=\nabla_{a} f+w \Upsilon_{a} f .
$$

On an oriented conformal $n$-manifold, the line bundle $\Lambda^{n}$ is also trivialised by a choice of metric. Specifically, we may use the inverse metric $g^{a b}$ to raise indices and then normalise the volume form $\epsilon_{b c \cdots d e}$ so that $\epsilon^{b c \cdots d e} \epsilon_{b c \cdots d e}=n$ !. Since $\widehat{g}^{a b}=\Omega^{-2} g^{a b}$ it follows that $\widehat{\epsilon}_{b c \cdots d e}=\Omega^{n} \epsilon_{b c \cdots d e}$ and hence that we may identify $\Lambda^{n}=L^{-n}$. It is easily confirmed that (7) implies

$$
\widehat{\nabla}_{a} \phi_{b c \cdots d e}=\nabla_{a} \phi_{b c \cdots d e}-n \Upsilon_{a} \phi_{b c \cdots d e}
$$

for $n$-forms $\phi_{b c \cdots d e}$, which is consistent with (9) for $w=-n$, as it should be. Combining (7) and (9) gives us a formula for the change of connection on $\Lambda^{1} \otimes L^{w}$, namely

$$
\widehat{\nabla}_{a} \phi_{b}=\nabla_{a} \phi_{b}+(w-1) \Upsilon_{a} \phi_{b}-\Upsilon_{b} \phi_{a}+\Upsilon^{c} \phi_{c} g_{a b}
$$


Let us now consider how the Laplacian $\Delta=\nabla_{a} \nabla^{a}$ acting on densities of weight $w$ is affected by a conformal rescaling of the metric. We compute:

$$
\widehat{\nabla}_{a} \widehat{\nabla}_{b} f=\nabla_{a} \widehat{\nabla}_{b} f+(w-1) \Upsilon_{a} \widehat{\nabla}_{b} f-\Upsilon_{b} \widehat{\nabla}_{a} f+\left(\Upsilon^{c} \widehat{\nabla}_{c} f\right) g_{a b}
$$

and so

$$
\begin{aligned}
\widehat{\Delta} f=\widehat{g}^{a b} \widehat{\nabla}_{a} \widehat{\nabla}_{b} f & =\Omega^{-2} g^{a b}\left(\nabla_{a} \widehat{\nabla}_{b} f+(w-1) \Upsilon_{a} \widehat{\nabla}_{b} f-\Upsilon_{b} \widehat{\nabla}_{a} f+\left(\Upsilon^{c} \widehat{\nabla}_{c} f\right) g_{a b}\right) \\
& =\Omega^{-2}\left(\nabla^{a} \widehat{\nabla}_{a} f+(n+w-2) \Upsilon^{a} \widehat{\nabla}_{a} f\right) \\
& =\Omega^{-2}\left(\nabla^{a}\left(\nabla_{a} f+w \Upsilon_{a} f\right)+(n+w-2) \Upsilon^{a}\left(\nabla_{a} f+w \Upsilon_{a} f\right)\right) .
\end{aligned}
$$

Here, we are using $g^{a b}$ to raise indices on the right hand side. Regrouping as

$$
\widehat{\Delta} f=\Omega^{-2}\left(\Delta f+(n+2 w-2) \Upsilon^{a} \nabla_{a} f+w\left(\nabla^{a} \Upsilon_{a}+(n+w-2) \Upsilon^{a} \Upsilon_{a}\right) f\right),
$$

we see that when $w=1-n / 2$ there are no first order derivatives in $f$ and

$$
\widehat{\Delta} f=\Omega^{-2}\left(\Delta f-(n / 2-1)\left(\nabla^{a} \Upsilon_{a}+(n / 2-1) \Upsilon^{a} \Upsilon_{a}\right) f\right) .
$$

On the other hand, the Riemann curvature tensor transforms by

$$
\widehat{R}_{a b c d}=\Omega^{2}\left(R_{a b c d}-\Xi_{a c} g_{b d}+\Xi_{b c} g_{a d}-\Xi_{b d} g_{a c}+\Xi_{a d} g_{b c}\right)
$$

where

$$
\Xi_{a b} \equiv \nabla_{a} \Upsilon_{b}-\Upsilon_{a} \Upsilon_{b}+\frac{1}{2} \Upsilon_{c} \Upsilon^{c} g_{a b}
$$

It follows immediately that the scalar curvature $R=g^{a c} g^{c d} R_{a b c d}$ transforms by

$$
\widehat{R}=\Omega^{-2}\left(R-2(n-1)\left(\nabla^{a} \Upsilon_{a}+(n / 2-1) \Upsilon^{a} \Upsilon_{a}\right)\right)
$$

From (10) and (11) we conclude that

$$
\widehat{\Delta} f-\frac{n-2}{4(n-1)} \widehat{R} f=\Omega^{-2}\left(\Delta f-\frac{n-2}{4(n-1)} R f\right) .
$$

If we also absorb the factor of $\Omega^{-2}$ into a change of conformal weight, then we conclude that $Y \equiv \Delta-\frac{n-2}{4(n-1)} R$ is a conformally invariant differential operator $Y: L^{1-n / 2} \rightarrow L^{-1-n / 2}$. This is the conformal Laplacian or Yamabe operator. The peculiar multiple of the scalar curvature that must be added to the Laplacian to achieve conformal invariance is often referred to a curvature correction term.

Now we are in a position to compare with the first order symmetries of the Laplacian on $\mathbb{R}^{n}$ found in Section 2. Of course, the curvature correction terms are absent for the flat metric on $\mathbb{R}^{n}$. Therefore, there is no difference between the Laplacian and the Yamabe operator provided we restrict our attention to conformal rescalings that take the flat metric to another flat metric. But flat-to-flat rescalings are precisely what are provided by the conformal Killing fields (5). Specifically, the conformal Killing fields are those vector fields whose flows preserve the conformal structure on $\mathbb{R}^{n}$ and, moreover, the flat-to-flat conformal factors generated in this way are general [2]. The conformal invariance of the Yamabe operator on a general conformal manifold therefore implies the invariance of the Laplacian on $\mathbb{R}^{n}$ under Lie derivative $\mathcal{L}_{V}$ for conformal Killing fields $V$. Here, as we have just seen, $\mathcal{L}_{V}$ should be interpreted as acting on the bundles $L^{1-n / 2}$ and $L^{-1-n / 2}$. At the end of Section 2 we found that the symmetry $\mathcal{D}_{V}$ given by (6) may be regarded as the Lie derivative on $\left(\Lambda^{n}\right)^{(n-2) / 2 n}$. These viewpoints now coincide because

$$
\Lambda^{n}=L^{-n} \Longrightarrow\left(\Lambda^{n}\right)^{(n-2) / 2 n}=L^{1-n / 2} .
$$

The same reasoning applies more generally. The geometric interpretation of conformal Killing fields combines with the conformal invariance of the Yamabe operator and we have proved: 
Theorem 1. Suppose that $V^{a}$ is a conformal Killing field on a Riemannian manifold, i.e. a solution of the equation (5). Then $\mathcal{D}_{V}$ given by (6) is a symmetry of the Yamabe operator. More precisely,

$$
\left(\Delta-\frac{n-2}{4(n-1)} R\right) \mathcal{D}_{V}=\delta_{V}\left(\Delta-\frac{n-2}{4(n-1)} R\right),
$$

where

$$
\mathcal{D}_{V}=V^{a} \nabla_{a}+\frac{n-2}{2 n}\left(\nabla_{a} V^{a}\right) \quad \text { and } \quad \delta_{V}=V^{a} \nabla_{a}+\frac{n+2}{2 n}\left(\nabla_{a} V^{a}\right) .
$$

In fact, it follows from proof of [10, Theorem 1] that these are the only symmetries of the Yamabe operator.

We have already observed that the differential pairing $\mathcal{D}_{V} f$ given by (6) is invariant in the very strong sense of being intrinsically defined on any manifold. Conformal invariance is a weaker statement but one that is easily verified directly. From (2) and (8),

$$
\widehat{\nabla}_{a} V^{b}=\nabla_{a} V^{b}+\Upsilon_{a} V^{b}-\Upsilon^{b} V_{a}+\Upsilon_{c} V^{c} \delta_{a}{ }^{b} \Longrightarrow \widehat{\nabla}_{a} V^{a}=\nabla_{a} V^{a}+n \Upsilon_{a} V^{a}
$$

whilst for $f$ a density of weight $w$ we have (9). It follows immediately that

$$
V^{a} \nabla_{a} f-\frac{w}{n}\left(\nabla_{a} V^{a}\right) f
$$

is a conformally invariant differential pairing and for $w=1-n / 2$ this agrees with (6).

\section{Higher order symmetries}

There are some second order symmetries of the Laplacian on $\mathbb{R}^{n}$ that should be regarded as trivial. If $\mathcal{D}=h \Delta$ for some smooth function $h$, then $\Delta \mathcal{D}=\delta \Delta$ simply by taking $\delta f=\Delta(h f)$. All symmetries preserve harmonic functions but $\mathcal{D}=h \Delta$ does this by dint of annihilating them. Ignoring these trivial symmetries, the second order symmetries of the Laplacian in $\mathbb{R}^{3}$ were found by Boyer, Kalnins, and Miller [3]. They add an extra 35 dimensions to the 11-dimensional space (3). In [10] all higher symmetries were found in all dimensions and, ignoring the trivial ones, there is an additional finite-dimensional space of second order ones. More precisely, there are second order symmetries

$$
\mathcal{D}_{V} f=V^{a b} \nabla_{a} \nabla_{b} f+\frac{n}{n+2}\left(\nabla_{a} V^{a b}\right) \nabla_{b} f+\frac{(n-2) n}{4(n+1)(n+2)}\left(\nabla_{a} \nabla_{b} V^{a b}\right) f
$$

for any trace-free symmetric tensor $V^{a b}$ satisfying

$$
\nabla_{a} V_{b c}+\nabla_{b} V_{c a}+\nabla_{c} V_{a b}=\frac{2}{n+2}\left(g_{a b} \nabla^{d} V_{c d}+g_{b c} \nabla^{d} V_{a d}+g_{c a} \nabla^{d} V_{b d}\right) .
$$

Such $V^{a b}$ are called conformal Killing tensors (of valence 2) and on $\mathbb{R}^{n}$ form a vector space of dimension

$$
\frac{(n-1)(n+2)(n+3)(n+4)}{12} .
$$

As detailed in [10], this is the 'additional space' of second order symmetries referred to above. As with first order symmetries (6), the pairing between valence 2 symmetric trace-free tensors $V^{a b}$ and densities $f$ of weight $1-n / 2$ given by the right hand side of (13) is conformally invariant under flat-to-flat conformal rescalings. Unlike the first order case, however, the pairing (13) is not invariant under general conformal rescalings of a general Riemannian manifold 
but, like the Laplacian, becomes so with the addition of suitable curvature correction terms. Specifically, for $V^{a b}$ any trace-free symmetric contravariant tensor and $f$ of weight $w$

$$
V^{a b} \nabla_{a} \nabla_{b} f-\frac{2(w-1)}{n+2}\left(\nabla_{a} V^{a b}\right) \nabla_{b} f+\frac{w(w-1)}{(n+1)(n+2)}\left(\nabla_{a} \nabla_{b} V^{a b}\right) f+\frac{w(n+w)}{(n+1)(n-2)} R_{a b} V^{a b} f,
$$

where $R_{a b}$ is the Ricci tensor, is a conformally invariant differential pairing on an arbitrary Riemannian manifold. When $w=1-n / 2$, we obtain

$$
\mathcal{D}_{V} f \equiv V^{a b} \nabla_{a} \nabla_{b} f+\frac{n}{n+2}\left(\nabla_{a} V^{a b}\right) \nabla_{b} f+\frac{(n-2) n}{4(n+1)(n+2)}\left(\nabla_{a} \nabla_{b} V^{a b}\right) f-\frac{n+2}{4(n+1)} R_{a b} V^{a b} f
$$

as a conformally invariant analogue of the flat symmetry operator (13). Whether this operator actually provides a symmetry of the Yamabe operator in general, however, is unclear. By analogy with Theorem 1, one might hope that if $V^{a b}$ were a conformal Killing tensor (14), then it would follow that

$$
\left(\Delta-\frac{n-2}{4(n-1)} R\right) \mathcal{D}_{V}=\delta_{V}\left(\Delta-\frac{n-2}{4(n-1)} R\right)
$$

for $\mathcal{D}_{V}$ as above and

$$
\delta_{V} f \equiv V^{a b} \nabla_{a} \nabla_{b} f+\frac{n+4}{n+2}\left(\nabla_{a} V^{a b}\right) \nabla_{b} f+\frac{n+4}{4(n+1)}\left(\nabla_{a} \nabla_{b} V^{a b}\right) f-\frac{n+2}{4(n+1)} R_{a b} V^{a b} f .
$$

This is currently unknown. The problem is that there does not seem to be any useful geometric interpretation of $V^{a b}$ being a conformal Killing tensor in parallel to $V^{a}$ being a conformal Killing field. In principle, the validity or otherwise of (16) should boil down to a calculation once the differential consequences of (14) are determined. Furthermore, the differential consequences of (14) can all be found by prolonging the system, as described in [5] for example. Nevertheless, it will surely be a difficult calculation.

\section{$5 \quad$ Further examples of invariant pairings}

The simple conformally invariant pairings we have found so far, namely (12) and (15), may be extended by allowing $V^{a}$ and $V^{a b}$ to have a general conformal weight as follows. It is convenient to set

$$
\Phi_{a b} \equiv \frac{1}{n-2}\left(R_{a b}-\frac{1}{n} R g_{a b}\right) .
$$

Proposition 1. Suppose that $V^{a}$ has conformal weight $v$ and $f$ has conformal weight $w$. Then

$$
(v+n) V^{a} \nabla_{a} f-w\left(\nabla_{a} V^{a}\right) f
$$

is conformally invariant. Suppose $V^{a b}$ is trace-free symmetric with conformal weight $v$ and $f$ has conformal weight $w$. Then

$$
\begin{gathered}
(n+v+2)(n+v+1) V^{a b} \nabla_{a} \nabla_{b} f-2(w-1)(n+v+1)\left(\nabla_{a} V^{a b}\right) \nabla_{b} f \\
+w(w-1)\left(\nabla_{a} \nabla_{b} V^{a b}\right) f+w(n+v+w)(n+v+2) \Phi_{a b} V^{a b} f
\end{gathered}
$$

is conformally invariant.

Proof. These are easily verified using the formulae for conformal rescaling developed in Section 3. 
Notice that there are several special cases occurring for particular values of $v$ and $w$. The form of (17) suggests setting $w=0$ or $v=-n$ and, when we do, we find

$$
(v+n) V^{a} \nabla_{a} f \quad \text { is invariant } \quad \forall V^{a} \Longrightarrow f \mapsto \nabla_{a} f \quad \text { is invariant }
$$

and

$$
-w\left(\nabla_{a} V^{a}\right) f \quad \text { is invariant } \quad \forall f \Longrightarrow V^{a} \mapsto \nabla_{a} V^{a} \quad \text { is invariant. }
$$

These are familiar invariant linear differential operators. The first one is the exterior derivative $d: \Lambda^{0} \rightarrow \Lambda^{1}$ and the second may be identified as $d: \Lambda^{n-1} \rightarrow \Lambda^{n}$. More interesting are the consequences of setting $w=1$ and $v=-n-1$ in (18). We obtain conformally invariant linear differential operators

$$
f \mapsto \nabla_{a} \nabla_{b} f-\frac{1}{n}(\Delta f) g_{a b}+\Phi_{a b} f \quad \text { and } \quad V^{a b} \mapsto \nabla_{a} \nabla_{b} V^{a b}+\Phi_{a b} V^{a b}, \quad \text { respectively. }
$$

The theory of conformally invariant linear differential operators is well understood $[1,4,12,14]$ and includes these two as simple examples. Other special values of the weights $v$ and $w$ show up as zeroes of the coefficients in (18). We have already seen that for $w=0$ there is an invariant differential operator $f \mapsto \nabla_{a} f$. Similarly, for $v=-n-2$ the operator $V^{a b} \mapsto \nabla_{a} V^{a b}$ is conformally invariant.

It is also interesting to note that, conversely, we can build the invariant pairing (18) from these various linear invariant operators. Specifically, if $V^{a b}$ has weight $v$ and $f$ has weight $w \neq 0$ then, at least where $f$ does not vanish,

$$
f^{-(n+v+1) / w} V^{a b} \text { has weight }-n-1 \quad \text { and } \quad f^{-(n+v+2) / w} V^{a b} \text { has weight }-n-2 .
$$

Therefore,

$$
\nabla_{a} \nabla_{b}\left(f^{-(n+v+1) / w} V^{a b}\right)+\Phi_{a b} f^{-(n+v+1) / w} V^{a b} \quad \text { and } \quad \nabla_{b}\left(f^{-(n+v+2) / w} V^{a b}\right)
$$

are invariant. If the second of these is multiplied by $f^{2 / w}$ we obtain a vector field of weight $-n$ and conclude that

$$
\nabla_{a}\left(f^{2 / w} \nabla_{b}\left(f^{-(n+v+2) / w} V^{a b}\right)\right)
$$

is also invariant. It follows, therefore, that the combination

$$
\begin{gathered}
w(n+v+2)(n+v+w) f^{(n+v+1+w) / w}\left(\nabla_{a} \nabla_{b}\left(f^{-(n+v+1) / w} V^{a b}\right)+\Phi_{a b} f^{-(n+v+1) / w} V^{a b}\right) \\
-w(n+v+1)(n+v+1+w) f^{(n+v+w) / w}\left(\nabla_{a}\left(f^{2 / w} \nabla_{b}\left(f^{-(n+v+2) / w} V^{a b}\right)\right)\right)
\end{gathered}
$$

is invariant. A short calculation reveals that this expression agrees with (18) and, although we supposed $w \neq 0$ and also that $f \neq 0$ in carrying out this derivation, the final conclusion is valid regardless. These tricks reveal some sort of relationship between invariant differential pairings and invariant linear differential operators, at least when one of the quantities to be paired is a conformal density.

These tricks are unavailable more generally but there are, nevertheless, many conformally invariant differential pairings. For example, if $V^{a b}$ is symmetric trace-free with conformal weight $v$ and $\phi_{a}$ has conformal weight $w$, then

$$
(n+v+2) V^{a b} \nabla_{a} \phi_{b}-(w-2)\left(\nabla_{a} V^{a b}\right) \phi_{b} .
$$

is invariant. Operators such as this one and (17) are referred to as first order, not only because there are no higher derivatives involved than first order but also because there are no cross terms 
of the form $\nabla V \bowtie \nabla \phi$ where $\bowtie$ denotes some algebraic pairing (we shall come back to this point soon). The theory of first order invariant differential pairings on a conformal manifold, or more generally on a manifold with AHS structure, is reasonably well understood thanks to recent work of Kroeske [16]. Following the approach of Fegan [14] in the conformal case and Cap, Slovák, and Souček [7] more generally, Kroeske classifies the first order invariant pairings provided that the weights of the tensors involved are so that invariant linear differential operators are excluded. This corresponds well to the relationship that seemed to be emerging in our examples. For higher order pairings, however, Kroeske [16] finds additional unexpected pairings and is able to present a satisfactory theory only in the flat projective setting.

\section{Some precise formulations}

When $E$ and $F$ are smooth vector bundles on some smooth manifold, it is well-known that linear differential operators $D: E \rightarrow F$ of order $\leq k$ are in 1-1 correspondence with vector bundle homomorphisms $J^{k} E \rightarrow F$ where $J^{k} E$ is the $k^{\text {th }}$ jet bundle of $E$. Indeed, it is usual to abuse notation and also write $D: J^{k} E \rightarrow F$, sometimes adopting this viewpoint as the definition of a linear differential operator. There are canonical short exact sequences of vector bundles

$$
0 \rightarrow \bigodot^{k} \Lambda^{1} \otimes E \rightarrow J^{k} E \rightarrow J^{k-1} E \rightarrow 0,
$$

where $\bigodot^{k} \Lambda^{1}$ is the bundle of symmetric covariant tensors of valence $k$ and the composition

$$
\bigodot^{k} \Lambda^{1} \otimes E \rightarrow J^{k} E \stackrel{D}{\longrightarrow} F
$$

is called the symbol of $D$. For further details, see [19] for example.

Differential pairings may be formulated similarly. Suppose another vector bundle $V$ is given and we wish to define what is a differential pairing $E \times F \rightarrow V$. The jet exact sequences (19) may be written as

$$
J^{k} E=E+\Lambda^{1} \otimes E+\bigodot^{2} \Lambda^{1} \otimes E+\bigodot^{3} \Lambda^{1} \otimes E+\cdots+\bigodot^{k} \Lambda^{1} \otimes E,
$$

meaning that $J^{k} E$ is filtered with successive quotients as shown. It follows that

$$
\begin{aligned}
& \bigodot^{2} \Lambda^{1} \otimes E \otimes F \\
& \Lambda^{1} \otimes E \otimes F \quad \oplus
\end{aligned}
$$

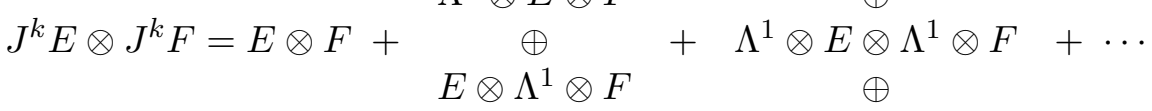

$$
\begin{aligned}
& E \otimes \bigodot^{2} \Lambda^{1} \otimes F
\end{aligned}
$$

and, following [16], we define the bi-jet bundle $J^{k}(E, F)$ as the quotient of $J^{k} E \otimes J^{k} F$ corresponding to the first $k+1$ columns of this composition series. There are bi-jet exact sequences starting with

$$
0 \rightarrow \begin{gathered}
\Lambda^{1} \otimes E \otimes F \\
E \otimes \Lambda^{1} \otimes F
\end{gathered} \rightarrow J^{1}(E, F) \rightarrow E \otimes F \rightarrow 0
$$

and

$$
\begin{aligned}
& \odot^{2} \Lambda^{1} \otimes E \otimes F \\
& \stackrel{\oplus}{\oplus} \Lambda^{1} \otimes E \otimes \Lambda^{1} \otimes F \rightarrow J^{2}(E, F) \rightarrow J^{1}(E, F) \rightarrow 0 . \\
& \stackrel{\oplus}{{ }^{2}} \otimes \odot^{1} \otimes F
\end{aligned}
$$


A differential pairing $E \times F \rightarrow V$ of order $k$ may now defined as a homomorphism of vector bundles $J^{k}(E, F) \rightarrow V$. Notice that it is the total order of the operator that is constrained to be less than of equal to $k$. For first order pairings, for example, we are excluding terms of the form $\nabla \psi \bowtie \nabla \phi$ (written in the presence of chosen connections). For second order pairings we exclude $\nabla^{2} \psi \bowtie \nabla \phi, \nabla \psi \bowtie \nabla^{2} \phi$, and $\nabla^{2} \psi \bowtie \nabla^{2} \phi$. The symbol of a pairing is defined as the composition

$$
\bigoplus_{j=0}^{k} \bigodot^{j} \Lambda^{1} \otimes E \otimes \bigodot^{k-j} \Lambda^{1} \otimes F \longrightarrow J^{k}(E, F) \longrightarrow V .
$$

Several other basic notions for linear differential operators immediately carry over to bilinear differential pairings. Suppose, for example, that we consider homogeneous vector bundles on a homogeneous space $G / P$. If $E$ and $F$ are induced from $P$-modules $\mathbb{E}$ and $\mathbb{F}$, then $J^{k}(E, F)$ will also be induced from a $P$-module, say $J^{k}(\mathbb{E}, \mathbb{F})$. If $V$ is induced from $\mathbb{V}$, a $G$-invariant differential pairing will correspond to a $P$-module homomorphism $J^{k}(\mathbb{E}, \mathbb{F}) \rightarrow \mathbb{V}$. In particular, when $G=\mathrm{SO}(n+1,1)$ and $G / P$ is the conformal sphere (as detailed in [9] for example) then these $P$-module homomorphisms correspond to flat-to-flat invariant differential pairings. This gives an approach to classification as adopted in [16]. Nevertheless, it remains a difficult problem.

\section{Other sources of invariant pairings}

Firstly, there are other conformally invariant differential operators whose symmetries give rise to invariant differential pairings. The square of the Laplacian is treated in [11] and the Dirac operator in [13]. There is also another source of pairings derived from the structure of the symmetry algebra as follows. Let us consider the symmetries of the Laplacian as in Sections 2 and 4. It is clear that the composition of symmetries is again a symmetry. In particular, if we compose two first order symmetries (6) on $\mathbb{R}^{n}$ then we find [10]

$$
\mathcal{D}_{V} \mathcal{D}_{W} f=\mathcal{D}_{V \odot W} f+\frac{1}{2} \mathcal{D}_{[V, W]} f-\frac{n-2}{4 n(n+1)}\langle V, W\rangle f+\frac{1}{n} V^{a} W_{a} \Delta f
$$

where $\mathcal{D}_{V \odot W}$ is given by (13) and

$$
\begin{aligned}
& (V \odot W)^{a b}=\frac{1}{2} V^{a} W^{b}+\frac{1}{2} V^{b} W^{a}-\frac{1}{n} g^{a b} V^{c} W_{c}, \\
& {[V, W]^{a}=V^{b} \nabla_{b} V^{a}-W^{b} \nabla_{b} V^{a},} \\
& \langle V, W\rangle=(n+2)\left(\nabla_{b} V^{a}\right)\left(\nabla_{a} W^{b}\right)-\frac{n+2}{n}\left(\nabla_{a} V^{a}\right)\left(\nabla_{b} W^{b}\right)-\frac{n+2}{n} V^{a} \nabla_{a} \nabla_{b} W^{b} \\
& \quad-\frac{n+2}{n} W^{a} \nabla_{a} \nabla_{b} V^{b}+\Delta\left(V_{a} W^{a}\right) .
\end{aligned}
$$

The last of these is constant when $V^{a}$ and $W^{a}$ are Killing fields on $\mathbb{R}^{n}$ and in this case coincides with their inner product under the Killing form in $\mathfrak{s o}(n+1,1)$. Remarkably, there is a curvature corrected version

$$
\begin{aligned}
& (n+2)\left(\nabla_{b} V^{a}\right)\left(\nabla_{a} W^{b}\right)-\frac{n+2}{n}\left(\nabla_{a} V^{a}\right)\left(\nabla_{b} W^{b}\right)-\frac{n+2}{n} V^{a} \nabla_{a} \nabla_{b} W^{b} \\
& \quad-\frac{n+2}{n} W^{a} \nabla_{a} \nabla_{b} V^{b}+\Delta\left(V_{a} W^{a}\right)-\frac{2(n+2)}{(n-2)} R_{a b} V^{a} W^{b}+\frac{2 n}{(n-1)(n-2)} R V_{a} W^{a}
\end{aligned}
$$

that provides an invariant differential pairing on arbitrary vector fields.

This approach to constructing invariant pairings is sometimes called quantisation: the symbol of an operator is specified, one attempts to build an invariant operator with this symbol, and then one composes these operators. Further examples are given by Duval and Ovsienko [8] in the conformal case and Fox [15] in the projective case.

Finally, there is a general construction of invariant pairings for very particular weights called cup products by Calderbank and Diemer [6]. This construction applies in any parabolic geometry but further discussion is beyond the scope of this article. 


\section{Acknowledgements}

It is a pleasure to acknowledge many extremely useful conversations with Vladimír Souček and Jens Kroeske. Support from the Australian Research Council is also gratefully acknowledged.

\section{References}

[1] Baston R.J., Eastwood M.G., Invariant operators, in Twistors in Mathematics and Physics, London Math. Soc. Lecture Note Ser., Vol. 156, Cambridge University Press, 1990, 129-163.

[2] Baird P., Wood J.C., Harmonic morphisms between Riemannian manifolds, Oxford University Press, 2003.

[3] Boyer C.P., Kalnins E.G., Miller W. Jr., Symmetry and separation of variables for the Helmholtz and Laplace equations, Nagoya Math. J. 60 (1976), 35-80.

[4] Branson T.P., Differential operators canonically associated to a conformal structure, Math. Scand. 57 (1985), 293-345.

[5] Branson T.P., Čap A., Eastwood M.G., Gover A.R., Prolongations of geometric overdetermined systems, Internat. J. Math. 17 (2006), 641-664, math.DG/0402100.

[6] Calderbank D.M.J., Diemer T., Differential invariants and curved Bernstein-Gelfand-Gelfand sequences, J. Reine Angew. Math. 537 (2001), 67-103, math.DG/0001158.

[7] Čap A., Slovák J., Souček V., Invariant operators on manifolds with almost Hermitian symmetric structures. III. Standard operators, Differential Geom. Appl. 12 (2000), 51-84, math.DG/9812023.

[8] Duval C., Ovsienko V., Conformally equivariant quantum Hamiltonians, Selecta Math. 7 (2001), 291-230.

[9] Eastwood M.G., Notes on conformal differential geometry, Rendi. Circ. Mat. Palermo Suppl. 43 (1996), $57-76$.

[10] Eastwood M.G., Higher symmetries of the Laplacian, Ann. Math. 161 (2005), 1645-1665, hep-th/0206233.

[11] Eastwood M.G., Leistner T., Higher symmetries of the square of the Laplacian, in Symmetries and Overdetermined Systems of Partial Differential Equations, IMA Volumes, no. 144, Springer Verlag, 2007, 319-338, math.DG/0610610.

[12] Eastwood M.G., Rice J.W., Conformally invariant differential operators on Minkowski space and their curved analogues, Comm. Math. Phys. 109 (1987), 207-228, Erratum, Comm. Math. Phys. 144 (1992), 213.

[13] Eastwood M.G., Somberg P., Souček V., Symmetries of the Dirac operator, in preparation.

[14] Fegan H.D., Conformally invariant first order differential operators, Quart. J. Math. 27 (1976), 371-378.

[15] Fox D.J.F., Projectively invariant star products, Int. Math. Res. Not. 2005 (2005), no. 9, 461-510, math.DG/0504596.

[16] Kroeske J., Invariant differential pairings, Acta Math. Univ. Comenian., to appear, math.DG/0703866.

[17] Miller W. Jr., Symmetry and separation of variables, Addison-Wesley, 1977.

[18] Penrose R., Rindler W., Spinors and space-time, Vol. 1, Cambridge University Press, 1984.

[19] Spencer D.C., Overdetermined systems of linear partial differential equations, Bull. Amer. Math. Soc. 75 (1969), 179-239. 